\title{
Tecnología, diversificación productiva y crecimiento: un modelo estructuralista ${ }^{1}$
}

\author{
Gabriel Porcile ${ }^{2}$ \\ Mario Cimoli ${ }^{3}$
}

\begin{abstract}
Resumo
O artigo apresenta um modelo Norte-Sul no qual a taxa de crescimento de longo prazo do Sul depende do aprendizado tecnológico e da mudança estrutural. Adota-se como ponto de partida o modelo de crescimento com restrição no Balanço de pagamentos, que permite identificar as condições necessárias para a convergência sustentável. Mostra-se que essa convergência requer que o cociente entre as elasticidades renda das exportações e das importações seja maior do que a unidade. Por sua vez, essas elasticidades são função do padrão de especialização. O modelo permite estudar como a interação entre a dinâmica do hiato tecnológico e da estrutura produtiva definem a taxa relativa de crescimento Norte-Sul e os salários relativos. Também são discutidos os efeitos da política e das instituições através de variações em alguns dos parâmetros mais importantes do modelo.
\end{abstract}

Palavras-chave: Macroeconomia; Desenvolvimento econômico; Inovações tecnológicas; Convergência (Economia).

\section{Abstract \\ Technology, productive diversification and growth: structuralist model}

The paper presents a North-South model in which the long run rate of growth of the South depends on technological learning and structural change. It takes as a point of departure the Balance-ofPayments-constrained growth model, which provides useful insights on the necessary conditions for sustained convergence. It is suggested that this requires that the ratio between the income elasticity of the demand for exports and imports to be higher than the unity. In turn, income elasticities are a function of the technology gap and of the patterns of specialization of the economy. The model allows for discussing how the interplay between the productive structure of the South and the dynamics of the technology gap shapes North-South relative rates of growth (and hence convergence or divergence) and relative wages in the long run. The paper also addresses the influence of policy and institutional variables by allowing key parameters in the model to vary.

Key words: Structuralist macroeconomics; Economic growth; Innovation and technologic diffusion; Conditional convergence.

JEL O41, O33, F43.

El objetivo de este artículo es presentar un marco teórico formal donde los temas de tecnología, estructura productiva y convergencia internacional puedan

(1) Trabalho recebido em março de 2007 e aprovado em julho de 2007.

(2) Departamento de Economía - Universidade Federal do Paraná (UFPR), Curitiba, Paraná, Brasil y CNPq. E-mail: <porcile@ufpr.br>.

(3) Universidad de Venecia e Cepal, Santiago de Chile. E-mail: <mario.cimoli@ cepal.org >.

Economia e Sociedade, Campinas, v. 16, n. 3 (31), p. 289-310, dez. 2007. 
ser analizados de una forma articulada desde la perspectiva estructuralista. Los elementos distintivos de dicha perspectiva surgen de combinar la teoría keynesiana del crecimiento impulsado por la demanda, basada en las elasticidades ingreso de la demanda de exportaciones e importaciones, con el estudio de los factores que definen las elasticidades en el largo plazo. Las mismas están asociadas a la evolución de la brecha tecnológica en el tiempo y a cómo interactúa con la estructura productiva. El modelo toma como punto de partida una economía internacional de dos países, Norte y Sur (o centro y periferia, respectivamente), con fuertes asimetrías tecnológicas y productivas, y analiza en qué condiciones dichas asimetrías tienden a reproducirse y a generar divergencia en los niveles de ingreso por habitante.

El artículo está estructurado en seis secciones, además de esta introducción y las conclusiones. La sección 1 presenta muy brevemente la teoría keynesiana del crecimiento con restricción externa. A partir de esta teoría, en la sección 2, se definen convergencia y divergencia internacional, como una función de las elasticidades ingreso de las exportaciones e importaciones. Se admite, en conformidad con las ideas estructuralistas, que dichas elasticidades reflejan la estructura productiva del Sur, y en particular su grado de diversificación hacia sectores intensivos en tecnología. La sección 3 presenta el modelo Ricardiano básico, a partir del cual se define el patrón de especialización internacional. Este modelo se amplía para incluir, en las secciones siguientes, la influencia de la dinámica de la brecha tecnológica (sección 4) y de los salarios relativos (sección 5). De esta forma se completa un modelo en el cual las dimensiones schumpeteriana y keynesiana se combinan para determinar la trayectoria de crecimiento del Sur. Finalmente, la sección 6 consiste de ejercicios de dinámica comparativa, discutiendo los efectos sobre la diversificación productiva de cambios en la política tecnológica o en el funcionamiento del mercado de trabajo. $\mathrm{Al}$ final del trabajo, un apéndice discute aspectos formales del modelo propuesto.

\section{Crecimiento con restricción externa: el modelo básico}

Se pretende desarrollar, a lo largo del artículo, un modelo que articule de forma coherente la dinámica Norte-Sur a partir de sus interacciones tecnológicas, productivas y comerciales. Para alcanzar ese objetivo es necesario avanzar por etapas. Primeramente se concentra la atención en sólo una de aquellas interacciones, a saber en cómo los flujos de comercio internacional afectan las tasas relativas de crecimiento a través de la necesidad de preservar, en el largo plazo, el equilibrio en cuenta corriente. Para ello se recurre a la teoría del crecimiento con restricción externa, que puede verse como una formalización, simple pero efectiva, de la perspectiva estructuralista. Inicialmente se presenta el modelo en su forma keynesiana "pura", en la que sólo actúan factores relativos a 
la demanda (las elasticidades ingreso de exportaciones e importaciones). Posteriormente tal perspectiva será complementada con la inclusión en el análisis de variables vinculadas a la oferta (tecnología y productividad).

El modelo keynesiano de crecimiento con restricción externa ya es ampliamente conocido y por esa razón se hará una exposición muy sumaria del mismo (Thirlwall y Hussein, 1982; McCombie y Thirlwall, 1994). ${ }^{4}$ Formalmente puede ser descrito por medio de las siguientes ecuaciones:

$$
\begin{aligned}
& \text { (1) } X=\left(\frac{P}{P^{*} E}\right)^{\phi}\left(Y^{*}\right)^{\mathcal{E}} \\
& \text { (2) } M=\left(\frac{P^{*} E}{P}\right)^{v} Y^{\pi} \\
& \text { (3) } P X+P F=P M \\
& \text { (4) } a(p+x)+(1-a)(p+f)=p+m \\
& \text { (5) } x=\phi\left(p-p^{*}-e\right)+\varepsilon y^{*} \\
& \text { (6) } m=v\left(p^{*}+e-p\right)+\pi y \\
& \text { (7) } y_{E}=\frac{(1+a \phi+v)\left(p-p^{*}-e\right)+a \varepsilon y^{*}+(1-a) f}{\pi}
\end{aligned}
$$

El modelo consta de dos países, que aquí se designan como Norte (cuyas variables se identifican con un asterisco) y Sur, siendo el Sur un país pequeño. $X$ es la cantidad exportada, $P$ el nivel de precios interno, $P^{*}$ el nivel de precios internacionales, $E$ la tasa nominal de cambio (unidades de la moneda nacional por unidad de moneda extranjera), $Y^{*}$ es el PIB del Norte, $M$ es la cantidad importada, $Y$ es el PIB del Sur y $F$ es la oferta real de recursos externos (denominados en moneda nacional). Los parámetros negativos $\phi$ y $v$ son elasticidades precio de la demanda de exportaciones e importaciones, respectivamente, mientras que los parámetros positivos $\varepsilon$ y $\pi$ son las elasticidades ingreso de las exportaciones e importaciones, respectivamente. Las letras minúsculas representan tasas de crecimiento en el tiempo (por ejemplo, $x=\frac{d X}{d t} \frac{1}{X} \equiv \frac{\dot{X}}{X}$ es la tasa de crecimiento de las cantidades exportadas), a no ser $a=P X /(P X+P F)$, que representa la participación de las divisas originadas por exportaciones en el total de divisas obtenidas por el país.

(4) Ver también los trabajos reunidos en Setterfield (2002). 
Las ecuaciones (1) y (2) son ecuaciones convencionales de demanda de exportaciones e importaciones, con elasticidades precio e ingreso constantes. La ecuación (3) impone una condición de equilibrio en balanza de pagos. Naturalmente, si $F=0$, ella se transforma en una condición de equilibrio en cuenta corriente. La ecuación (4) expresa el equilibrio en balanza de pagos en términos dinámicos, lo que requiere la igualdad entre la tasa de crecimiento del valor de las exportaciones (en moneda nacional) y la tasa de crecimiento del valor de las exportaciones más la tasa de crecimiento de la entrada de capitales (estas dos últimas variables ponderadas por sus respectivas participaciones en el total de divisas). Naturalmente, si $a=1$, no hay entrada de capitales y la ecuación dinámica de equilibrio se refiere sólo a la cuenta corriente. Las ecuaciones (5) y (6) representan tasas de crecimiento de las cantidades exportadas e importadas. La ecuación (7) surge de usar las ecuaciones (5) y (6) en la condición dinámica de equilibrio dada por la ecuación (4). Se obtiene así la tasa de crecimiento de la economía compatible con el equilibrio en balanza de pagos, designada como $y_{E}$.

Si se desconsidera la posibilidad de recurrir a préstamos externos $(a=1){ }^{5}$ se obtiene una nueva ecuación para la tasa de crecimiento con equilibrio externo:

$$
\text { (8) } y_{E}=\frac{(1+\phi+v)\left(p-p^{*}-e\right)+\varepsilon y^{*}}{\pi}
$$

La ecuación (8) indica que la tasa de crecimiento del Sur en el largo plazo depende de la tasa de crecimiento del Norte, $y^{*}$, de la tasa de desvalorización de la tasa de cambio real $\left(p-p^{*}-e\right)$, de las elasticidades precio de la demanda de exportaciones e importaciones $(\phi$ e $v)$, y de las elasticidades ingreso de las exportaciones e importaciones $(\varepsilon$ y $\pi$ ). Si la condición de Marshall-Lerner es válida, entonces $|\phi+v|>1$ y la desvalorización real del tipo de cambio tendrá un efecto positivo sobre la tasa de crecimiento de la economía Sur.

\section{Convergencia sostenible en el modelo con restricción externa}

En esta sección el modelo de crecimiento con restricción externa será usado para derivar de manera simple las condiciones en las cuales sería posible la convergencia internacional. Con este objetivo se supondrá que el principio de la paridad del poder de compra se verifica en el largo plazo. Bajo esta condición la tasa de cambio real fluctuará en torno a su valor de equilibrio y puede admitirse

(5) El mismo resultado se obtiene si se imagina que la economía del Sur no puede crecer con base en un endeudamiento creciente. Los flujos positivos en un período deben compensarse por flujos negativos de capital en otros. En ese sentido la ecuación (8) no es contradictoria con el flujo de capitales. Una discusión más profunda del tema puede encontrarse en Thirlwall y Houssein (1982) y Moreno-Brid (1988-89). Sobre los límites del crecimiento con endeudamiento externo, ver Bresser-Pereira y Nakano (2003). 
que $\left(p-p^{*}-e\right)=0$. De ello se deriva una versión simplificada de la ecuación (8), que se expresa en las siguientes ecuaciones:

$$
\begin{aligned}
& \text { (9) } y_{E}=\frac{\varepsilon y^{*}}{\pi} \\
& \text { (10) } y_{E}=\frac{x}{\pi}
\end{aligned}
$$

Obsérvese que (10) toma en cuenta que, si el tipo de cambio es estable, entonces es verdad que $\varepsilon y *=x$ (ver ecuación (5)), donde $x$ es la tasa de crecimiento de la cantidad exportada en el tiempo. La ecuación (9), a su vez, puede ser reescrita como una ecuación de convergencia / divergencia internacional:

$$
\text { (11) } \frac{y_{E}}{y^{*}}=\frac{\varepsilon}{\pi}
$$

En efecto, el Sur crecerá a una tasa más elevada que el Norte (convergencia) si la elasticidad ingreso de sus exportaciones supera la elasticidad ingreso de las importaciones.

La relación entre tasas de crecimiento y elasticidades ingreso, que se fundamenta en la necesidad de mantener el equilibrio externo en el largo plazo, es una regularidad empírica muy robusta. La misma representa lo que la literatura keynesiana denomina "Ley de Thirlwall", y la literatura de inspiración más convencional denomina "Regla de 45" (Krugman, 1989). A pesar de que los distintos autores están de acuerdo en la validez empírica de la ecuación (11), difieren en cuanto a sus fundamentos teóricos, en particular con relación al papel que juegan las fuerzas de la oferta y la demanda en el ajuste de la tasa de crecimiento efectiva $(y)$ a la tasa de crecimiento con equilibrio externo $\left(y_{E}\right)$. Es bastante consensual, sin embargo, la idea de que el progreso técnico y la estructura productiva tienen un papel muy significativo en el comportamiento de las elasticidades. Para Krugman, éstas siempre responden de modo endógeno, exactamente en la proporción necesaria para que la tasa de crecimiento de la economía iguale la tasa de crecimiento de la productividad total de los factores. El modelo de Thirlwall, en cambio, concuerda en que la estructura productiva contribuye, del lado de la oferta, a definir los valores de las elasticidades ingreso (como, además, lo sostiene la tradición estructuralista ${ }^{6}$ ), pero no admite que sean un mero reflejo de lo que ocurre con la productividad total de los factores. Progreso técnico y elasticidades interactúan de formas menos previsibles y automáticas que en el modelo de Krugman.

(6) Cf. Prebisch $(1949,1981)$ y Rodríguez (1980). 
En este trabajo se toma como punto de partida la visión keynesiana de Thirlwall y se avanza en la discusión de los factores que contribuyen a configurar las elasticidades ingreso de las exportaciones e importaciones. Más específicamente, se supondrá que la relación $\varepsilon / \pi$ es una función monotónica creciente del grado de diversificación de la estructura productiva hacia sectores más intensivos en tecnología. Dicha diversificación puede ser representada por un índice $z$, que corresponde al cociente entre el número de bienes que se producen en el Sur y el número total de bienes transados en la economía internacional. Por el momento no será discutida la dinámica de z, pero sí interesa reescribir (11) explicitando que el cociente de las elasticidades es función de la estructura $z$ :

$$
\text { (12) } \frac{y_{E}}{y^{*}}=\frac{\varepsilon}{\pi}(z)
$$

A partir de la ecuación (12) es posible construir un diagrama describiendo las condiciones necesarias para la convergencia internacional sostenible (ver Figura 1).

Figura 1

Convergencia, divergencia y la Regla de $45^{\circ}$

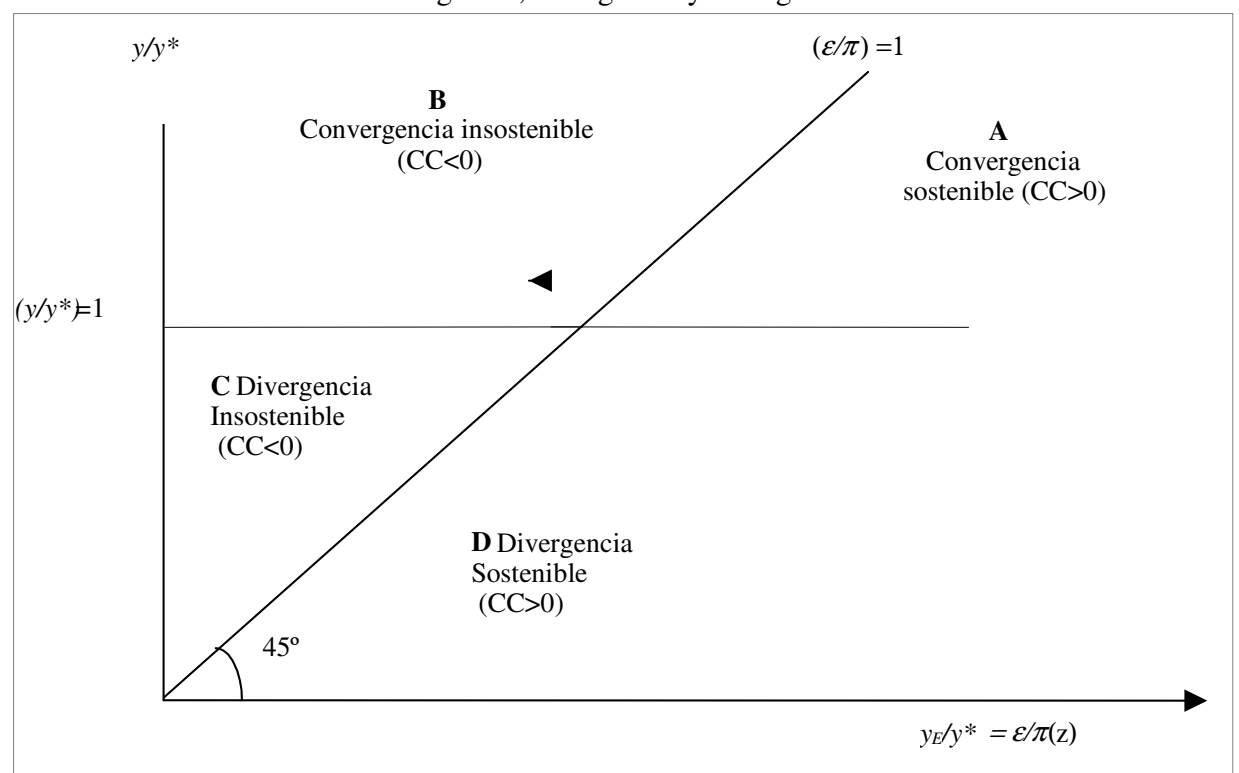

Nota: En el eje de las ordenadas se representa la tasa relativa de crecimiento efectivo y en el de las abscisas la tasa relativa de crecimiento con equilibrio externo. La línea de $45^{\circ}$ indica todos los puntos en que la tasa efectiva y la tasa de equilibrio coinciden. La tasa de equilibrio depende del comportamiento de las elasticidades ingreso de las exportaciones y las importaciones, que son función de la estructura productiva (z). Los puntos por encima de la línea horizontal $y / y^{*}=1$ son de convergencia internacional, y los puntos debajo de la línea de $45^{\circ}$ son sostenibles en el largo plazo (saldo comercial positivo).

$\mathrm{A}=$ Convergencia sostenible

$\mathrm{B}=$ Convergencia insostenible (deuda)

$\mathrm{C}=$ Divergencia y déficit (deuda)

D = Divergencia y superávit (pago de deuda, exportación de capital o acumulación de reservas). 
La Figura 1 muestra en el eje de las ordenadas la tasa relativa de crecimiento efectiva Sur / Norte $\left(y / y^{*}\right)$ y en el eje de las abscisas la tasa relativa de crecimiento con equilibrio externo $\left(y_{E} / y^{*}=\varepsilon / \pi(z)\right)$. La línea de $45^{\circ}$ representa los puntos en los que coinciden la tasa efectiva y la tasa de equilibrio, para los cuales el saldo en cuenta corriente es igual a cero. Cuando la tasa efectiva $y / y^{*}$ supera la unidad, habrá convergencia, ya que la economía del Sur crece a una tasa más alta que la del Norte. Pero no todos los puntos de convergencia son sostenibles, sino sólo aquellos que no generan saldo comercial negativo. Los puntos arriba de la línea de $45^{\circ}$ son puntos de crecimiento no sostenibles en el largo plazo porque suponen endeudamiento creciente. La región de convergencia sostenible, por lo tanto, es la región $\mathrm{A}$, formada por los puntos que obedecen a la siguiente condición:

$$
\text { (13) } \frac{\varepsilon}{\pi} \geq \frac{y}{y^{*}}>1
$$

En la región B la convergencia no es sostenible: en algún momento la deuda tendrá que pagarse o el financiamiento externo se volverá más caro o más escaso, por lo que la economía será obligada a ajustarse y a crecer de acuerdo con la restricción externa. Las regiones $\mathrm{C}$ y D son especialmente preocupantes. En la región $\mathrm{C}$ el país no consigue alcanzar la convergencia internacional, ni siquiera con recurso al endeudamiento externo. Su competitividad es tan débil (a la que corresponde una brecha tecnológica elevada y una estructura productiva poco dinámica) que cualquier incremento significativo en la tasa de crecimiento produce inmediatamente desequilibrios que la desestabilizan y frenan. Finalmente, en el caso D, el país crece a tasas menores a las compatibles con su ya baja relación $\varepsilon / \pi$. En este caso, el mismo puede estar pagando deudas contraídas en un período anterior, lo que lo obliga a transferir recursos al exterior, o puede estar acumulando reservas con el objetivo de prevenir algún tipo de vulnerabilidad externa o ataque especulativo. No es tampoco una situación deseable, ya que en ella el Sur no explota plenamente su potencial de crecimiento.

En resumen, las tasas de crecimiento relativo del Sur y del Norte (y por lo tanto la posibilidad de convergencia internacional), compatibles con el equilibrio externo, son una función de las elasticidades ingreso de las exportaciones e importaciones del Sur. A su vez, éstas últimas dependen del grado de diversificación productiva alcanzado por el país. En las próximas secciones se analiza como la innovación y la difusión internacional de tecnología interactúan con la estructura productiva para definir su grado de diversificación. 


\section{El modelo Ricardiano de especialización internacional: aspectos básicos}

En esta sección el foco recae sobre los determinantes de la especialización internacional. Se admite que la misma es función de las dinámicas tecnológica y del cambio estructural, pero aún no se discute qué factores gobiernan estas dinámicas. La sección debe verse, por lo tanto, como una etapa en la construcción de un modelo más amplio.

Una forma simple de abordar la relación entre diversificación productiva, tecnología y convergencia es tomando como punto de partida el modelo Ricardiano de comercio internacional. Primeramente se hará una breve exposición de la estructura básica del modelo original propuesto por Dornbusch et al. (1976). En la sección siguiente se lo extiende para poder incluir los efectos de la brecha tecnológica, del patrón de especialización y de la competencia imperfecta sobre el crecimiento.

Se asume que la economía mundial está formada por un continuo de bienes $i=0 \ldots N$ y dos países, el Norte (tecnológicamente avanzado) y el Sur (rezagado). La economía funciona en condiciones de competencia perfecta, donde cada empresa produce un cierto tipo de bien contratando un único factor de producción, el trabajo. Cada bien es producido con un número de horas de trabajo igual a $a_{i}$ en el Sur y $a^{*}{ }_{i}$ en el Norte. La variable $A_{i}=a^{*}{ }_{i} / a_{i}$ representa la productividad relativa del Sur con respecto a la del Norte. Cuanto mayor (menor) es $A_{i}$, mayor (menor) es la ventaja comparativa del Sur. Es posible ordenar los bienes $i=0$ hasta $i=N$ en una secuencia creciente con la ventaja comparativa del Norte, de modo que el bien número cero es aquél para el cual la ventaja comparativa del Sur es máxima y el bien número $N$ es aquél para el cual la ventaja comparativa del Norte es máxima. Ello define una curva de productividades relativas $A A$ negativamente inclinada con relación al número de bienes producidos en el Sur. Se define, además, una variable $z=n / N$, igual a la relación entre el número de bienes producidos en el Sur $(n)$ y el número total de bienes existente $(N)$. Naturalmente, la suma de los bienes producidos en el Sur $(n)$ y el Norte $(n *)$ debe ser igual al total $N$, por lo que $z$ sólo puede variar entre cero y la unidad $(o \leq z \leq 1)$. A su vez, la curva $A A$ es negativamente inclinada con relación a $z$ por la propia manera en que fue construido el índice $z$.

En competencia perfecta el precio de un bien se determina por el costo unitario de producción:

$$
\begin{aligned}
& \text { (14) } P_{i}=w a_{i} \\
& \text { (15) } P *_{i} E=w^{*} E a^{*}{ }_{i}
\end{aligned}
$$


donde $w$ es el salario nominal, $E$ la tasa de cambio nominal (unidades de la moneda del Sur por unidad de la moneda del Norte) y $P_{i}$ el precio del bien. El asterisco denota variables y parámetros que corresponden al país Norte. Un bien será producido en el Sur si su precio es igual o más bajo que en el Norte, lo que implica $w a_{i} \leq w^{*} E a^{*}{ }_{i}$. Más adelante se discuten los determinantes de las variables $a$ y $w$. Reordenando términos y recordando que $W=w /\left(w^{*} E\right)$ y que $A=$ $a * / a$, se tiene que el bien será producido en el Sur si se cumple la siguiente condición:

$$
\text { (16) } A_{i} \geq W
$$

Determinar el patrón de especialización de cada país requiere conjugar la curva de productividades relativas con la de costos relativos. La curva $W=w /$ $w^{*} E$ representa el salario nominal relativo del Sur $(w)$ con respecto al del Norte $\left(w^{*} E\right)$, medidos en la moneda del Sur. La relación $W$ aumenta con el número de bienes producidos en el Sur (suponiendo que la elasticidad de oferta de mano de obra no sea infinita) debido al efecto positivo que el aumento de la producción ejerce sobre la demanda de trabajo. A medida que se diversifica la economía del Sur, ésta obtendrá, concomitantemente, aumentos en el nivel de empleo y de salarios relativos.

Puede verse en la Figura 2 cómo se determina el patrón de especialización a partir de los costos relativos de producción. El Sur produce los bienes desde $z=$ 0 hasta alcanzar un cierto valor crítico $z_{c}$, a partir del cual el costo de producción se vuelve más bajo en el Norte.

La diversificación productiva del Sur puede obtenerse por medio del aumento de la productividad relativa o de la caída del salario relativo. Imagínese por un momento que el Sur reduce las diferencias de productividad, por ejemplo, a través de una mejora de sus capacidades tecnológicas con respecto al Norte $(+\Delta A$, en la Figura 1): la curva $A A$ se moverá hacia la derecha, hasta alcanzar la posición $A^{\prime} A$ '. Como resultado, el Sur habrá cambiado su patrón de inserción externa, siendo capaz de producir un conjunto mayor de bienes con un salario relativo más alto. Este proceso representa lo que Fajnzylber (1990) denomina una mejora en la competitividad "auténtica" - aumento de la participación del Sur en los mercados externo e interno, al que acompaña un incremento del salario relativo. Inversamente, una reducción del salario relativo $(-\Delta W)$, sin cambios en la productividad, también permite diversificar la estructura productiva, al trasladar hacia abajo la curva $W W$, pero ello ocurre a expensas de una reducción del salario relativo en el Sur (competitividad "espuria” en la denominación de Fajnzylber). 
Figura 2

El Modelo Ricardiano

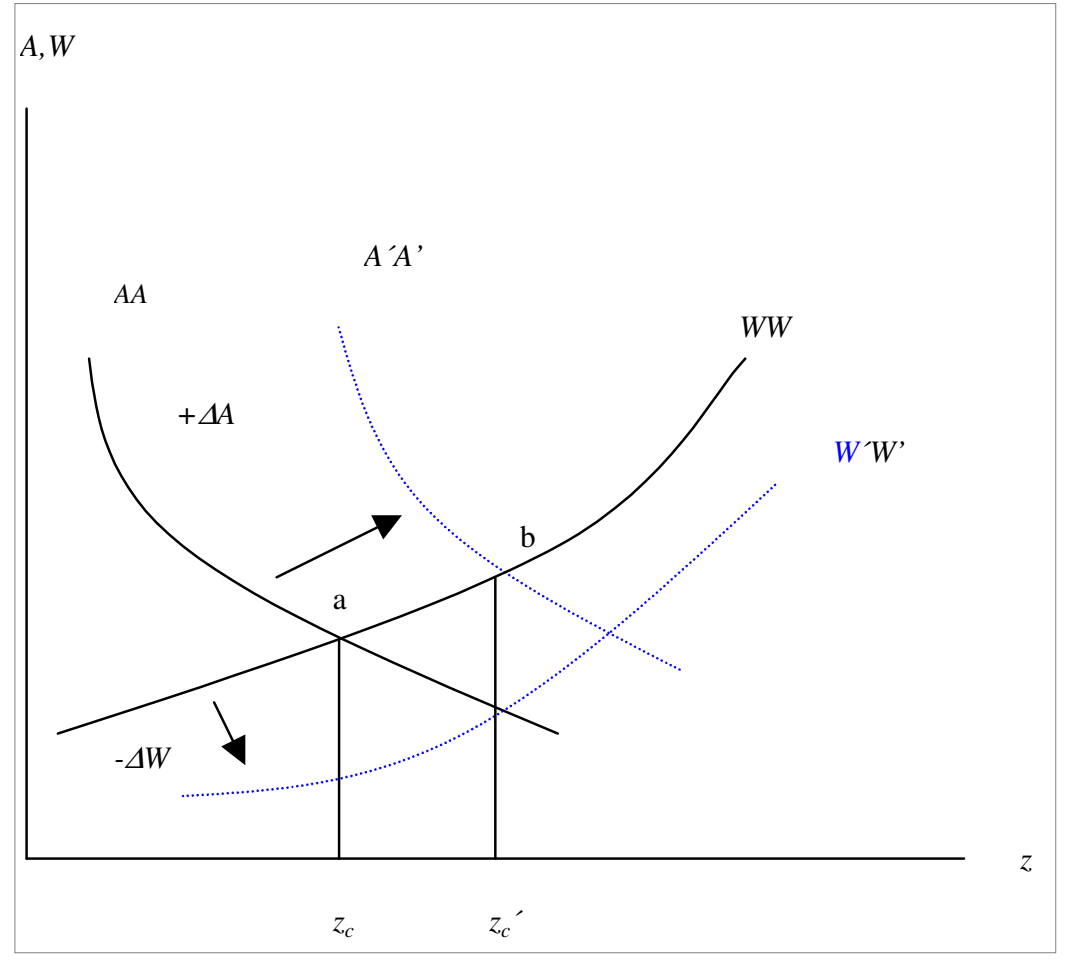

Debe observarse, además, que el impacto de las alteraciones en el salario relativo sobre la especialización depende de la inclinación de $A A$, que define el multiplicador de la brecha tecnológica (Cimoli, 1988). Si esta inclinación no es muy alta, pequeños cambios en el salario relativo producen cambios importantes en la especialización. Inversamente, un alto valor del multiplicador de la brecha (una curva $A A$ muy inclinada) implica que variaciones en el salario relativo no tendrán el poder de modificar substancialmente la competitividad externa de la economía.

En la próxima sección se presenta un modelo formal en que las interrelaciones entre cambio estructural, progreso técnico, comercio y crecimiento son exploradas más rigurosamente tomando como punto de partida el modelo Ricardiano. El mismo se modifica substancialmente, al incluir en él el análisis de los determinantes de las asimetrías tecnológicas, del salario relativo en competencia imperfecta y de las tasas relativas de crecimiento, que constituyen temas centrales en la perspectiva estructuralista. 


\section{La dinámica de la Brecha}

Entender los cambios en el patrón de especialización requiere especificar la dinámica de las curvas $A A$ y $W W$. En esta sección se propone un modelo sumamente simplificado, un prototipo de fácil manipulación a partir del cual se puede entender mejor las interrelaciones entre progreso técnico, especialización y crecimiento. Al mismo tiempo, se extienden los resultados encontrados en Cimoli (1988) y Dosi et al (1990).

¿De qué depende la productividad relativa con que cada bien es producido? Se argumenta que la misma es función de dos variables. La primera es la brecha tecnológica $(G)$, definida como la relación entre el acervo de de conocimientos en el Norte $\left(T_{N}\right)$ con respecto al mismo acervo en el Sur $\left(T_{S}\right)$. Formalmente:

$$
\text { (17) } G=\frac{T_{N}}{T_{S}}
$$

donde $0<G \leq 1$. Cuanto más alto es el valor de la brecha, menor será $A_{i}=a^{*}{ }_{i} / a_{i}$ para todo $i$ (i.e. menor será la productividad relativa del Sur con respecto al Norte).

La segunda variable que afecta el comportamiento de la productividad relativa, para un dado valor de la brecha, es la intensidad tecnológica con que cada bien se produce. Se supone que el país tecnológicamente rezagado es relativamente más eficiente en bienes de menor intensidad tecnológica que en los de mayor intensidad tecnológica. Siendo así, el ranking de productividades relativas representando por la curva $A(z)$ corresponde al ranking de intensidad tecnológica en la producción de los distintos bienes $z$. La pendiente negativa de la curva $A A$ refleja la dificultad creciente del Sur en imitar, adaptar y usar de forma eficiente tecnologías que se vuelven más complejas a medida que se diversifica la estructura productiva, para un cierto valor de la brecha de conocimientos entre los dos países. Es esa dificultad creciente la que explica el aumento de la diferencia de productividades pari passu con $\mathrm{z}$.

Las consideraciones anteriores pueden expresarse formalmente por medio de la siguiente ecuación:

$$
\text { (18) } A=\gamma-\alpha G-\beta z(\operatorname{curva} A A)
$$

En la ecuación (4), cambios en la brecha tecnológica $G$ se traducen en cambios en la posición de la curva $A A$. El parámetro $\alpha$ traduce el efecto de la brecha tecnológica sobre las productividades relativas, mientras que el parámetro $\beta$ muestra como éstas responden al aumento en la intensidad tecnológica de los bienes. El supuesto implícito es que un cambio en la brecha afecta la 
productividad relativa de todos los bienes en exactamente la misma proporción, por lo que la inclinación de $A A$ no sufriría cambios. El movimiento hacia arriba de la curva $A A$, en la Figura 2 (como resultado de la mejora en las productividades relativas), expresa justamente el efecto de una disminución de la brecha tecnológica Norte-Sur.

Como la brecha afecta la posición de la curva $A A$ y a través de ella al patrón de especialización, su magnitud y evolución son muy importantes para entender la especialización y el crecimiento.¿Qué factores afectan la brecha? Hay una oferta bastante amplia de teorías acerca de la dinámica tecnológica en modelos Norte-Sur. En general, éstas admiten que la brecha inicial ofrece un potencial de imitación y de aprendizaje que el Sur, bajo ciertas condiciones, podría aprovechar. Dos situaciones se distinguen con respecto al efecto que la brecha inicial ejerce sobre la evolución de la brecha en el tiempo (Fagerberg, 1988; Verspagen, 1993; Patel y Pavitt, 1998; Narula, 2004):

a)En la primera situación el país Sur muestra un rezago tecnológico tan grande con respecto a los países avanzados que no es siquiera capaz de imitar la tecnología generada en el Norte. Las oportunidades de imitación existen, pero el Sur no dispone de la base mínima de capacidades necesaria para beneficiarse de ellas. En este caso, cuánto más alta es la brecha inicial, más rápidamente el Sur quedará atrás en la carrera tecnológica.

b) Una segunda situación es cuando el Sur ya sobrepasó el nivel crítico de capacidades necesarias para dar lugar al catching up. En este caso, cuanto mayor es la brecha en el momento inicial, más rápido el Sur aprende y más rápidamente la brecha se cierra (disminuye) en el tiempo. A diferencia de lo que ocurre en el caso (a), la brecha representa más una oportunidad que una desventaja insalvable.

En términos formales, la primera situación implica que la tasa de variación de la brecha en el tiempo depende positivamente de la brecha inicial. En el segundo caso, la tasa de variación de la brecha depende negativamente del nivel inicial.

Pero el cambio de la brecha no depende sólo de la brecha inicial. Una segunda variable que afecta la evolución de la brecha en el tiempo es la propia estructura productiva. Ésta es un componente muy importante de la perspectiva estructuralista, que puede resumirse así: algunos sectores, principalmente aquellos intensivos en tecnología, son capaces de promover procesos de aprendizaje tecnológico, de generar externalidades y de difundir innovaciones más fuertemente que otros (Nelson and Pack, 1999; Ros, 2000; Peneder, 2002; Cimoli et al, 2005; Hausmann et al, 2005). Por eso el peso de los mismos en la estructura productiva es un determinante importante de la capacidad de aprendizaje de la economía y afectará, por esa razón, la evolución de la brecha en el tiempo. 
Combinando las dos variables anteriores (brecha inicial y estructura productiva) se obtiene la siguiente ecuación que describe la evolución en el tiempo de la brecha tecnológica Norte-Sur:

$$
\text { (19) } \hat{G}=\frac{d\left(T_{N} / T_{S}\right)}{d t} \frac{T_{S}}{T_{N}}=a-c G-b z
$$

Donde $\hat{G}=\dot{G} / G$ es la tasa de cambio de la brecha $G=T_{N} / T_{S}$ en el tiempo y $z=(n / N)$ es la proporción del total de los bienes que se produce en el Sur. El parámetro $b$ depende de los retornos crecientes en el aprendizaje tecnológico, asociados a externalidades, learning by doing y learning by using, así como al estímulo a la inversión en nuevas generaciones de bienes de capital. Un alto valor de dicho parámetro indica que cuanto menos diversificado es el Sur (menor el peso de los sectores intensivos en tecnología, lo que corresponde a un bajo valor de $z$ ), más rápidamente éste quedará atrás en la carrera tecnológica con el Norte.

El parámetro $c$, a su vez, depende de la capacidad del Sur de aprovechar el potencial de imitación propiciado por la brecha $G$. Se ha supuesto en la ecuación que $c$ es positivo y ello quiere decir que el Sur ya alcanzó el nivel mínimo de capacidades necesario para aprender con la tecnología del Norte. ${ }^{7}$ El valor del parámetro $c$ es una función de las inversiones en ciencia y tecnología en el Sur, de su sistema de educación, del acervo de capital humano (Nelson y Phelps, 1966), y de las instituciones que promueven la difusión de conocimientos. En términos generales, refleja aquél conjunto de variables que la literatura schumpeteriana designa genéricamente como Sistema Nacional de Innovación.

Además de la tecnología, la especialización depende también de los salarios relativos, de los que se ocupa la próxima sección.

\section{La dinámica de los salarios y la especialización}

Los factores que gobiernan los salarios relativos concurren para definir la especialización. Este es el punto principal abordado en esta sección. Como en la anterior, el modelo propuesto se aparta significativamente del modelo Ricardiano original. Las diferencias surgen de un conjunto específico de supuestos que se enumeran en el ítem A3 del Apéndice y que, de forma muy sintética, se presentan a continuación.

En primer lugar, el salario relativo depende de la brecha tecnológica. Ello se explica porque una brecha menor conlleva una más alta productividad relativa.

(7) Formalmente, $c<0$ si $G>G m$ y $c>0$ si $G<G$, donde $G m$ representa el valor de la brecha a partir del cual ya no es posible la imitación. 
A medida que la distancia tecnológica se acorta, los trabajadores del Sur esperan que también decline la diferencia entre sus salarios y los del Norte. En tales circunstancias, los sindicatos juzgarán que tienen una probabilidad más alta de recibir aumentos, ya que ellos no comprometerían la competitividad internacional, dado el mark-up.

La segunda variable que afecta el nivel de los salarios relativos es la diversificación productiva del Sur. Al aumentar el número $z$ de bienes que el Sur produce, se expande la producción y se estimula la demanda de trabajado en dicho país. Como consecuencia, bajo los supuestos de que la oferta de trabajo no es perfectamente elástica, $W$ será una función creciente de $z$. Formalmente:

$$
\text { (20) } W=-u G+v z(\operatorname{curva} W W)
$$

Con mark-up fijo en el Norte y el Sur, la especialización de equilibrio ocurre en el punto donde se cortan las curvas $A A$ y $W W(A=W)$. En él se respetan exactamente las ventajas comparativas de cada país en el comercio internacional y no habrá razones para que la producción de algún bien migre de un país a otro. Tómese como ejemplo el caso en que la productividad relativa del Sur supera la curva de salarios relativos $(A>W)$. Existe en ese caso un estímulo para que se trasladen actividades del Norte al Sur, donde los costos son menores, de tal forma que $A$ cae y $W$ aumenta, hasta que se alcanza el equilibrio. Paralelamente hay un proceso de cambio estructural según el cual el Sur pasa a producir un mayor número de bienes (aumenta $z$ ), incorporando sectores de mayor intensidad tecnológica. Formalmente:

$$
\text { (21) } \hat{z}=\zeta(A A-W W)
$$

donde $\zeta>0$ es un coeficiente de ajuste, que, sin pérdida de generalidad, en lo restante del trabajo será supuesto igual a la unidad.

Usando en (7) las ecuaciones (4) y (6) se obtiene:

$$
\text { (22) } \hat{z}=\gamma-\alpha G-\beta z+u G-v z
$$

Y reordenado términos:

$$
\text { (23) } \hat{z}=\gamma-f z-g G
$$

donde $f=\beta+v$, y $g=u-\alpha$.

donde $\alpha>u$. Las ecuaciones (19) y (23) representan un sistema de ecuaciones diferenciales que permiten encontrar endógenamente los valores de $z$ y $G$. El lector encontrará en un Apéndice matemático al final del capítulo el análisis detallado de las condiciones de estabilidad y equilibrio del sistema. A continuación, se ilustrará gráficamente las consecuencias sobre la brecha y la diversificación productiva de cambios en los parámetros del modelo. 


\section{Dinámica comparativa: política industrial, negociación salarial y competitividad internacional}

En esta sección se procede del siguiente modo. Se encuentran las isoclinas correspondientes a las ecuaciones diferenciales (19) y (23). Posteriormente se muestra como éstas varían cuando choques provocados por la política económica o por el arreglo institucional en el mercado de trabajo alteran los parámetros estructurales del modelo. El objetivo es indagar sobre las implicaciones de política que surgen del modelo en dos dimensiones centrales para las economías del Sur, a saber: la necesidad del catching-up tecnológico y los desafíos de integrar una fuerza de trabajo que aún permanece, en gran medida, en sectores informales o de baja productividad.

Igualando la ecuación (19) a cero se obtiene todas las combinaciones de $G$ y $z$ para las que la brecha tecnológica es constante en el tiempo (curva $G G$, donde $\hat{G}=0$ ).

(24) $G=\frac{a}{c}-\frac{b}{c} z(\operatorname{curva} G G)$

Igualando la ecuación (23) a cero se obtienen todas las combinaciones de $G$ y $z$ para las que la estructura productiva se mantiene estable (curva $Z Z$, donde $\hat{z}=0)$.

(25) $G=\frac{\gamma}{g}-\frac{f}{g} z($ curva $Z Z)$

El punto de corte de las curvas $G G$ y $Z Z$ (dadas por las ecuaciones (5') y $\left.\left(8^{\prime}\right)\right)$ representa un equilibrio en el sentido de que $G$ y $z$ son constantes en ese punto. Los valores de equilibrio de $z$ y $G$ son los siguientes:

$$
\begin{aligned}
& \text { (26) } z^{*}=\frac{\chi c-a g}{c f-b g} \\
& \text { (27) } G^{*}=\frac{a f-\not b}{c f-b g}
\end{aligned}
$$

Como se muestra en el Apéndice, el sistema formado por las ecuaciones (19) y (23) es estable, bajo ciertos supuestos.

A partir de los resultados anteriores es posible realizar algunos ejercicios de dinámica comparativa, en los que cambios exógenos en los parámetros del modelo representan los efectos de decisiones de política, del cambio en el marco institucional o de una nueva correlación de fuerzas en la negociación salarial. Se 
supondrá dos tipos de choques: en el primero, el gobierno implementa una política más activa de difusión tecnológica; en el segundo, los sindicatos encuentran condiciones de negociación menos favorables que en el pasado.

¿Qué ocurre si el Sur adopta una nueva política tecnológica que fortalece su capacidad de aprendizaje y de imitación de la tecnología del Norte (por ejemplo, ampliando las inversiones en educación o el apoyo directo e indirecto a las inversiones en I+D)? La nueva política se reflejará en una caída del parámetro $a$ o en aumentos de los parámetros $b$ y c. En la Figura $3 \mathrm{~A}$ se representa el caso más simple, el de la caída de $a$. Básicamente, una reducción de ese parámetro implica que el Sur eleva sus gastos autónomos en I+D en mayor proporción que el Norte. La curva $G G$ se traslada hacia $G G^{\prime}$, mejoran las capacidades tecnológicas del Sur frente al Norte y la brecha tecnológica de equilibrio cae en relación a la brecha inicial $\left(G_{2}<G_{1}\right)$. Como resultado, la economía del Sur se diversifica, iniciando la producción de un mayor número de bienes que antes $\left(z_{2}>z_{1}\right)$. Todo ello ocurre en consonancia con el aumento del salario relativo, como puede apreciarse en la ecuación (6) ( $W$ aumenta cuando $G$ cae y $z$ aumenta, dados $u$ y $v$ ), ya que los aumentos en la productividad relativa y el empleo favorecen el salario relativo del Sur (ver el Apéndice al final del trabajo).

Figura 3A

El efecto de la política tecnológica (aumento de $a$ )

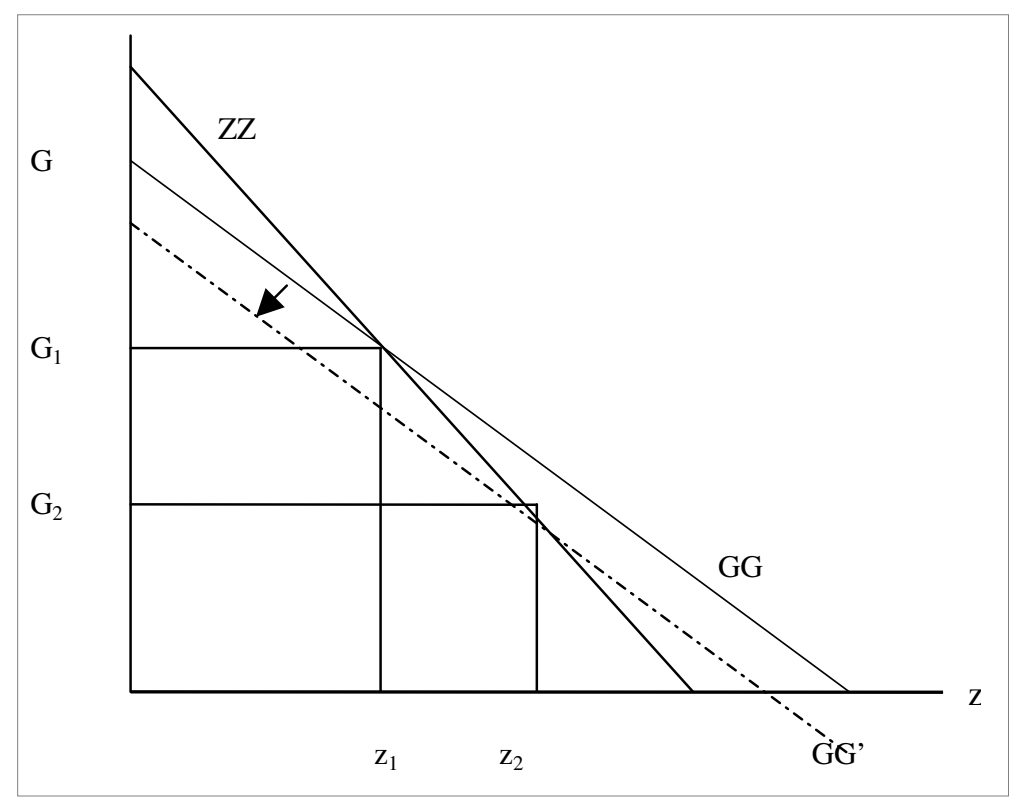


Figura 3B

Elasticidades y diversificación productiva

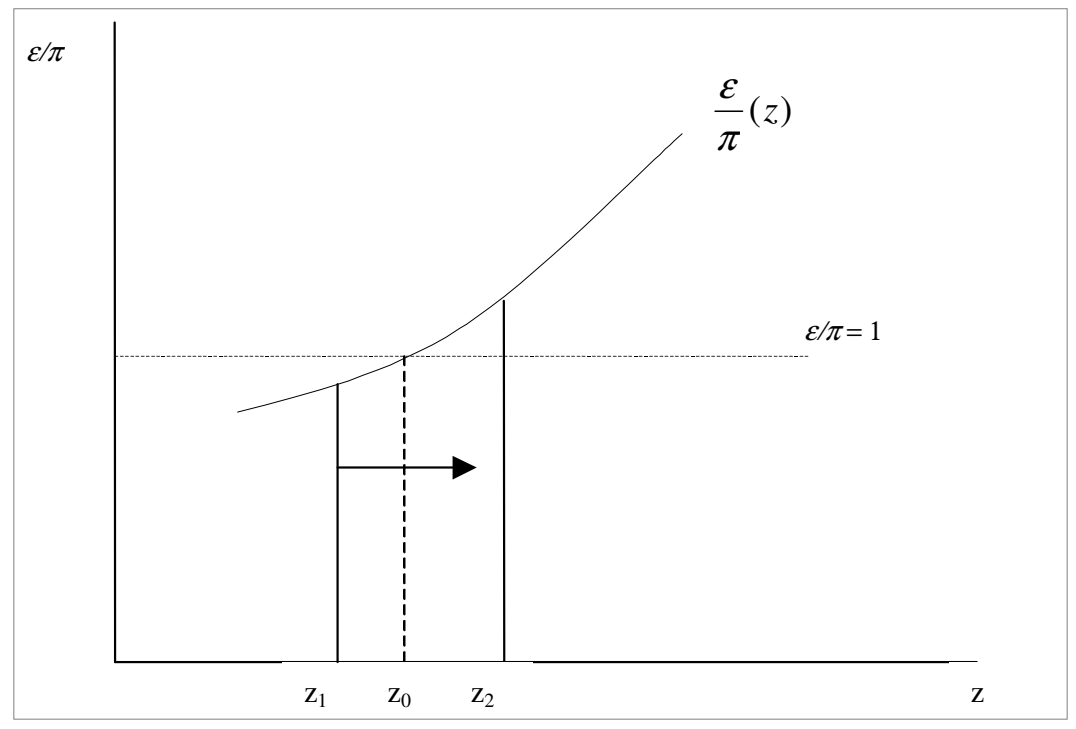

Figura 3C

Efecto de un cambio en el mercado laboral (caída de $v$ )

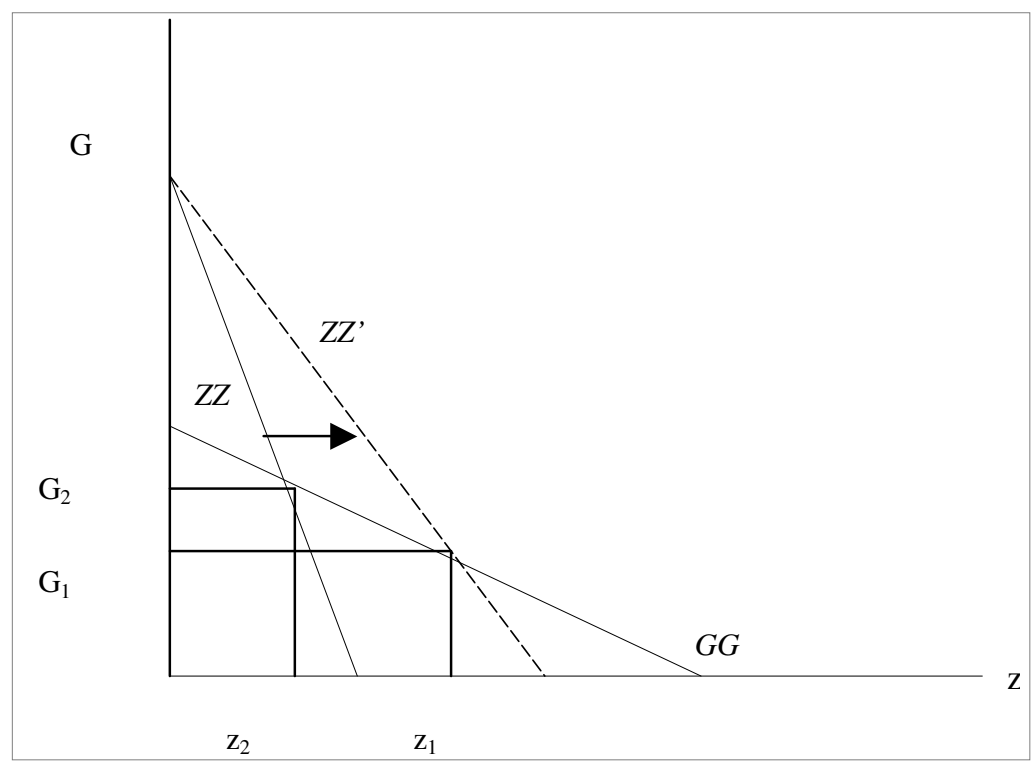

Nota: En la Figura 3A se representa el caso de una nueva política tecnológica que induce un aumento de los gastos autónomos en I+D en el Sur. La brecha cae, el salario relativo aumenta y la diversificación productiva lograda se refleja en el aumento de la razón entre las elasticidades $\varepsilon / \pi$ (que es una función de $z$ ) en la Figura 3B, posibilitando una tasa más elevada de crecimiento en el Sur. En el ejemplo, el Sur pasa de una situación de divergencia $(\varepsilon / \pi<1)$ a una de convergencia $(\varepsilon / \pi>1)$. Un efecto similar se obtiene si hay un cambio en el ambiente de negociación salarial en el Sur que reduce inicialmente el salario relativo, como representado en la figura $3 \mathrm{C}$. Mientras tanto, si no hay retornos crecientes a la diversificación productiva, en este segundo caso el salario relativo del Sur podría no recuperarse de su caída inicial. 
La Figura 3B muestra el efecto de la diversificación productiva sobre la razón entre las elasticidades ingreso $\varepsilon / \pi$. En el ejemplo se supuso que $z$ era inicialmente $z_{1}<z_{0}$, siendo $z_{0}$ la diversificación productiva para la cual el Norte y el Sur crecen exactamente a la misma tasa $(\varepsilon / \pi=1$; ver Figura 1$)$. La nueva política tecnológica permite que el Sur diversifique su estructura hasta $z_{2}$, lo que implica $\varepsilon / \pi>1$. De acuerdo con la condición (13), la economía podría entonces salir de una situación de divergencia para alcanzar la convergencia sostenible.

¿Qué ocurre si hay un cambio, no en la política tecnológica, sino en las condiciones en que funciona el mercado de trabajo? Más específicamente, se supone que en el momento cero cae $v$ (y por lo tanto $f$ ), sea porque las expectativas sindicales se vuelven más pesimistas acerca de la oferta de empleo en el futuro, sea porque las instituciones que regulan el mercado de trabajo se vuelven desfavorables a los trabajadores. La caída de $v$ implica un menor salario relativo en el Sur para un dado valor de $G$ y $z$, lo que a su vez provoca que algunas actividades se trasladen desde el Norte hacia el Sur. La consecuente diversificación productiva del Sur genera una tasa mayor de aprendizaje tecnológico y una reducción de la brecha tecnológica. Ambos movimientos tienen un efecto positivo sobre el salario relativo. Gradualmente éstos se elevan en el Sur, mientras disminuyen los estímulos al aprendizaje generados por la brecha, hasta que se alcanza un nuevo equilibrio, con un valor más alto de $z$ y más bajo de $G$. Muy probablemente, a lo largo de ese proceso, el salario relativo del Sur habrá aumentado. El resultado paradojal (una reducción de la capacidad de los trabajadores de apropiarse de los aumentos de productividad produce un salario real más elevado) se explica porque existen retornos crecientes en el aprendizaje tecnológico $(b>0)$. El aumento inicial de competitividad (cae el salario relativo con mark-up constante) hace posible la diversificación que, en secuencia, tiene efectos fuertes sobre el empleo y la productividad.

Obsérvese, mientras tanto, que el modelo no excluye la posibilidad de que la reducción del poder de negociación sindical sólo genere competitividad espuria, ${ }^{8}$ lo que ocurre si se diversifica la economía al costo de una reducción del salario relativo. Ello ocurre cuando $c(\beta+v)>b(u+g)+c v$ (ver el Apéndice al final del trabajo). Tal desigualdad se verifica inmediatamente en el caso específico en que no hay retornos crecientes en el aprendizaje frente a la diversificación productiva $(b=0)$. En otras palabras, en una economía en la que la diversificación ocurre hacia actividades de bajo contenido tecnológico (que generan pocas

8 Nótese que competitividad espuria se define aquí de una manera más restrictiva que en Fajnzylber (1990). Este último autor considera competitividad espuria la que se vincula con una reducción de salarios reales. En este trabajo corresponde a una reducción del salario relativo nominal. El salario real crecerá en función de los aumentos de productividad en los dos polos, aun cuando el salario nominal se mantenga constante. 
externalidades hacia el resto del sistema), los efectos positivos del aumento de la competitividad internacional no se traducen en convergencia salarial.

\section{Conclusiones}

En este trabajo fue presentado un modelo de inspiración estructuralista en que la dimensión keynesiana del crecimiento (representada por la relación entre las elasticidades ingreso de la demanda de exportaciones e importaciones) se complementa con el análisis de la influencia de la tecnología y la estructura productiva sobre dichas elasticidades. El trabajo también discute los factores que afectan la dinámica del cambio estructural y del aprendizaje tecnológico, entre ellos la política tecnológica y las condiciones de negociación salarial. Ejercicios de dinámica comparativa sugieren que una política tecnológica más activa favorece al mismo tiempo los salarios relativos y la convergencia. Por otro lado, una disminución del poder de negociación de los sindicatos favorece la diversificación y la convergencia, pero puede reducir el salario relativo del Sur si los retornos crecientes del aprendizaje frente a la diversificación son muy débiles.

Si bien en equilibrio la brecha permanece constante, en la medida que ésta afecta las elasticidades, su nivel es capaz de influencia las tasas de crecimiento económico en el Sur y el Norte. Una baja diversificación productiva implica que la razón de las elasticidades será inferior a la unidad y por lo tanto habrá divergencia de ingresos por habitante en el largo plazo entre el Norte el Sur.

\section{Bibliografía}

BRESSER-PEREIRA, L. C.; NAKANO, Y. Crescimento econômico com poupança externa? Revista de Economia Política, v. 23, n. 2 (90), p. 1-25, abr./jun. 2003.

CIMOLI, M. Technological gaps and institutional asymmetries in a North-South Model with a continuum of goods. Metroeconomica, 39, p. 245-274, 1988.

CIMOLI, M.; PORCILE, G.; PRIMI, A.; VERGARA, S. Cambio estructural, heterogeneidad productiva y tecnología en América Latina. In: CIMOLI, M. (Ed.). Heterogeneidad estructural, asimetrías tecnológicas y crecimiento en América Latina. Santiago de Chile: Cepal/BID, 2005.

DOSI, G.; PAVITT, K.; SOETE, L. Technology and international trade. Edgar Elgar, 1990.

FAGERBERG, J. Why growth rates differ. In: DOSI, G.; FREEMAN, C., SILVERBERG, G.; SOETE, L. (Ed.). Technical change and economic theory. London: Cambridge University Press, 1988.

FAJNZYLBER, F. Industrialización en América latina: de la caja negra al casillero vacio. Cuadernos de la Cepal, Santiago de Chile, CEPAL, 60, 1990.

HAUSMANN, R.; HWANG, J.; RODRIK, D. What you export matters. John F. Kennedy School of Government, Harvard University, Dic. 2005. (Working Paper, RPW05-063). 
Gabriel Porcile / Mario Cimoli

KRUGMAN, Paul. Differences in income elasticities and secular trends in real exchange rates. European Economic Review, May 1989.

MCCOMBIE, J. S. L.; THIRLWALL, A. P. Economic growth and balance of payments constraint. New York: St Martin's Press, 1994.

MORENO-BRID. O capital inflows and the balance-of-payments constrained growth model. Journal of Post-Keynesian Economics, v. 21, n. 2, p. 283-298, winter 1998-99.

NARULA, R. Understanding absorptive capacities in an innovation systems context: consequences for economic and employment growth. DRUID Working Paper, n. 04-02, Dec. 2004.

NELSON, R.; PACK, H. The Asian miracle and modern growth theory. The Economic Journal, Royal Economic Society, Blackwell Publishers, 109, p. 416-436, 1999.

; PHELPS, E. Investments in human, technological diffusion and economic growth. American Economic Review; 61, p. 69-75, 1966.

PATEL, P.; PAVITT, K. Uneven (and divergent) technological accumulation among advanced countries: evidence and a framework of explanation. In: DOSI, G.; TEECE, D. J.; CHYTRY, J. Technology, organization and competitiveness. Oxford University Press, 1998.

PENEDER, M. Tracing empirical trails of Schumpeterian development. Papers on Economics and Evolution, Max Planck Institute for Research in Economic Systems, Viena, 2002.

PREBICH, R. El desarrollo económico de América Latina y sus principales problemas. Comisión Económica para América Latina, 1949. (Documento E/CN 12.89).

Económica, 1981.

Capitalismo periférico: crisis y transformación. México: Fondo de Cultura

ROS, J. Development theory and the economics of growth. Ann Arbor: The University of Michigan Press, 2000.

RODRÍGUEZ, O La teoría del subdesarrollo de la Cepal. Mexico: Fondo de Cultura Económica, 1980. (8. ed., 1993).

SETTERFIELD, M. The economics of demand-led growth. Challenging the supply-side vision of the long run. Cheltenham: Edward Elgar, 2002.

THIRLWALL, A. P.; HUSSAIN, M. N. The balance of payments constraint, capital flows and growth rates differences between developing countries. Oxford Economic Papers, 10, p. 498-509, 1982.

VERSPAGEN, B. Uneven growth between interdependent economies. London: Avebury, 1993. 


\section{Apéndice}

\section{A1 Estabilidad del Sistema}

El sistema está formado por dos ecuaciones diferenciales, la (19) y la (23).

(19) $\hat{G}=a-c G-b z$

(23) $\hat{z}=\gamma-g G-f z$

La traza y el determinante del sistema son los siguientes:

(A1) Traza: $-c-f$

(A2) Determinante: $c f-g b$

El sistema es estable si $g$ es negativo o si $c f>g b$, supuesto adoptado en el artículo. Es interesante notar que si la economía del Sur no hubiera alcanzado aún el nivel crítico necesario para la imitación, y el parámetro $c$ fuera negativo $(G>G m)$, el equilibrio sería inestable (un repulsor o un punto de silla).

Deben imponerse, además, las restricciones adicionales $a f>\not b$ y $\chi>a g$, necesarias para que los valores de equilibrio de $G$ y $z$ sean números positivos (véase las ecuaciones (26) y (27)).

\section{A.2 Efectos de los cambios en a y $v$ sobre el salario relativo $W$}

Para analizar los efectos de cambios en $a$ y $v$ sobre los salarios relativos en equilibrio debe calcularse la derivada parcial de $W$ con relación a aquellos parámetros cuando $z$ y $G$ están en equilibrio, esto es, cuando:

$$
\text { (A3) } W=-u G+v z=-u\left(\frac{a f-\not b}{c f-b g}\right)+v\left(\frac{\gamma c-a g}{c f-b g}\right)
$$

La derivada parcial de (A3) con relación a $a$ será, sin ambigüedades, un número negativo:

$$
\text { (A4) } \frac{\delta W}{\delta a}=-\frac{(\gamma-a g)(u b+c v)}{(c f-b g)^{2}}
$$

Así, una caída de $a$, fruto de un mayor esfuerzo tecnológico en el Sur, genera un aumento del salario relativo. Ya la derivada parcial de $W$ con respecto a $v$ tiene signo indeterminado:

$$
\text { (A5) } \frac{\delta W}{\delta v}=\frac{(\not c-a g)(c f-b g-u b-c v)}{(c f-b g)^{2}}
$$

El signo de esta derivada será positivo si $c f>b(g+u)+c v$. En tal caso la pérdida de poder de negociación de los trabajadores (caída de $v$ ) produce una caída del 
salario relativo. Esta situación obviamente se obtiene si $b=0$, ya que entonces para que la derivada parcial sea positiva es necesario que $c f>c v$, condición que siempre se cumple porque $f \equiv \beta+v$ (siendo $\beta$ un número positivo). Pero si son fuertes los retornos crecientes a la diversificación productiva, la caída de $v$ inicia un proceso de aprendizaje que resulta en un aumento del salario relativo del Sur. En ese caso, una caída del poder de negociación sindical generaría, vía retornos crecientes, no sólo la diversificación productiva, sino también el aumento del salario relativo del Sur en equilibrio.

\section{A.3 Supuestos sobre lucros, precios y salarios}

Existe competencia imperfecta en el Norte y el Sur. Por simplicidad, se asume que en el Norte salarios nominales son fijos, aunque el resultado no cambia si se supone que los salarios crecen a una tasa constante. El mark-up es constante y el mismo en el Norte y el Sur. La tasa de progreso técnico y el aumento de la productividad son constantes y uniformes en los distintos sectores. Ello ocurre a pesar de cada sector contribuye de forma diferente al aprendizaje tecnológico. La razón de ello es que dicha contribución asume la forma de una externalidad que favorece por igual toda la estructura productiva. De esa forma la tasa de inflación en el Norte será:

$$
\text { (A6) } \hat{P}^{*}=\hat{a}^{*}
$$

En el Sur los salarios se definen por medio de negociaciones entre sindicatos y firmas para generar el salario relativo previsto por la ecuación (20). Como los salarios nominales son constantes en el Norte y el salario relativo en equilibrio también lo es, entonces los salarios nominales serán constantes en el Sur en equilibrio. Siendo así, será verdad que:

$$
\text { (A8) } \hat{P}-\hat{P}^{*}=\hat{a}-\hat{a}^{*}
$$

Como en equilibrio la brecha es constante y la tasa de progreso técnico es la misma en el Norte y el Sur, las tasas externa e interna de inflación serán las mismas.

Vale la pena resaltar que en equilibrio no hay diferencias en las tasas de crecimiento de lucros y salarios entre el Norte y el Sur, pero sí en las tasas de crecimiento del producto. Eso se explica porque la estructura del Norte, más diversificada, tiene una elasticidad ingreso de la demanda más alta por los bienes que produce, como lo muestra la ecuación (12). Por ello, su economía es más estimulada que la del Sur por la expansión de la demanda internacional. Las diferencias entre las tasas de crecimiento se traducen en ese contexto en un mayor desempleo en el Sur y en migración de trabajadores desde el Sur hacia el Norte. Tal resultado es coherente con la persistencia de problemas de heterogeneidad en la estructura del empleo en el Sur, aunque el tema no es abordado en este trabajo (ver, por ejemplo, Ros, 2000, cap. 2; Cimoli et al., 2005). 